opportunity to adopt appropriate antibiotic prescribing behaviors. ${ }^{7}$ In contrast, the summary TPB scores did not correlate with antibiotic prescribing behavior. This finding contrasts with a systematic review of TPB domain scores reporting an association with antibiotic prescription behaviors. ${ }^{8}$ It is plausible that the weight of the individual TPB determinants requires future refinement. ${ }^{8} \mathrm{~A}$ second study finding was the key predictor of "considering patients as first priority" as a key predictor of appropriate antibiotic use. This finding suggests a patient safety and quality-improvement opportunity, while additional efforts may exist to minimize unnecessary antibiotic combinations for surgical prophylaxis and to shorten postoperative antibiotic duration.

The limitations of this study include acknowledgment of reported findings which may not be generalizable to other study populations, given the exploratory study design, small sample size, and single institutional study site. Additionally, despite structured interviews, inherent bias may have occurred in the TTM and TPB assessments, and have influenced the unweighted, cumulative TPB scores. Future work is planned for assessment of TTM stage-based prescriber interventions associated with antibiotic prescribing practices along with further characterization of the TPB intrapersonal behavior theory.

Supplementary material. To view supplementary material for this article, please visit https://doi.org/10.1017/ice.2019.167.

Author ORCIDs. Anucha Apisarnthanarak (iD 0000-0001-6390-9519

Financial support. No financial support was provided relevant to this article.
Conflicts of interest. Linda M. Mundy is a full-time employee at American Regent, Inc. and this work was conducted without compensation and independently of this employment. All other authors report no conflict of interest relevant to this article.

\section{References}

1. Sartelli M, Kluger Y, Ansaloni L, et al. A global declaration on appropriate use of antimicrobial agents across the surgical pathway. Surg Infect (Larchmt) 2017;18:846-853.

2. Prochaska JO, Velicer WF. The transtheoretical model of health behavior change. Am J Health Promot 1997;12:38-48.

3. Ajzen I. The theory of planned behavior. Organ Behav Hum Decis Process 1991;50:179-211.

4. Apisarnthanarak A, Eiamsitrakoon T, Mundy LM. Behavior-based interventions to improve hand hygiene adherence among intensive care unit healthcare workers in Thailand. Infect Control Hosp Epidemiol 2015;36:517-521.

5. Tamma PD, Miller MA, Cosgrove SE. Rethinking how antibiotics are prescribed: incorporating the 4 moments of antibiotic decision making into clinical practice. JAMA 2019;321:139-140.

6. Kunin CM, Tupasi T, Craig WA. Use of antibiotics. A brief exposition of the problem and some tentative solutions. Intern Med 1973;79:555-560.

7. Charani E, Edwards R, Sevdalis N, et al. Behavior change strategies to influence antimicrobial prescribing in acute care: a systematic review. Clin Infect Dis 2011;53:651-662.

8. Warreman EB, Lambregts MMC, Wouters RHP, et al. Determinants of in-hospital antibiotic prescription behaviour: a systematic review and formation of a comprehensive framework. Clin Microbiol Infect 2019;25: $538-545$.

\title{
Dentists' perceptions of antimicrobial use for dental procedures
}

\author{
Yasuhiro Kano MD ${ }^{1}$, Yasuaki Tagashira MD², , Daisuke Kobayashi DDS, $\mathrm{PhD}^{3}$ and Hitoshi Honda MD, $\mathrm{PhD}^{1}$ (1) \\ ${ }^{1}$ Division of Infectious Diseases, Tokyo Metropolitan Tama Medical Center, Tokyo, Japan, ${ }^{2}$ Department of Microbiology, Juntendo University Graduate School of \\ Medicine, Tokyo, Japan and ${ }^{3}$ Department of Dentistry and Oral Maxillofacial Surgery, Tokyo Metropolitan Tama Medical Center, Tokyo, Japan
}

To the Editor-The importance of promoting antimicrobial stewardship in dentistry is being increasingly recognized; up to $10 \%$ of all antimicrobials are prescribed by dentists in high-income countries, ${ }^{1,2}$ and a previous study revealed that only $8.2 \%$ of antimicrobial prophylaxes for dental procedures were appropriate. ${ }^{3}$ Although professional societies widely recommend antimicrobial prophylaxis against infective endocarditis (IE) among high-risk populations, ${ }^{4,5}$ no recommendations exist for antimicrobial prophylaxis against local infections or complications following invasive dental procedures, including tooth extraction and dental implant placement. Recent systematic reviews have revealed that antimicrobial prophylaxis can prevent local infections and other complications due to these procedures. ${ }^{6,7}$

Although understanding dentists' perceptions of antimicrobial use and prescribing patterns is essential to promoting antimicrobial stewardship in the discipline, these perceptions are still poorly

Author for correspondence: Liling Tang MB, Yijishan Hospital of Wannan Medical College, 2 West Zheshan Rd, 241001, Anhui Province, China. Email: yjsygk2017@sina.com

Cite this article: Zhang P and Tang L. (2019). Current status of infection control professionals in a Chinese city. Infection Control \& Hospital Epidemiology, 40: 1081-1083, https://doi.org/10.1017/ice.2019.186 understood. The aim of the study was to investigate dentists' perception of antimicrobial use to promote antimicrobial stewardship in the field.

\section{Material and Methods}

An online questionnaire on antimicrobial use before and after a dental procedure was administered to regional dental conference attendees (Japanese Society of Oral and Maxillofacial Surgeons, JSOMS) in December 2018. In total, 54 close-ended survey questions were used to collect data, including current patterns of antimicrobial prophylaxis against IE, infections and complications following tooth extraction or dental implant surgery, and dentists' perceptions of antimicrobial use.

\section{Results}

Of the 231 dentists attending the session, 111 (48.1\%) responded to the questionnaire. Moreover, $70 \%$ of respondents were male, with the median age of 36 years (range, 24-64 years) and median postgraduate duration of 11 years (range, $1-44$ years). University hospitals were the most common place of employment $(n=49,44.1 \%)$ (Appendix 1 online). 
Table 1. Dentists' Perceptions of Antimicrobial Prophylaxis Against Infective Endocarditis and Infection or Complications After Selected Dental Procedures

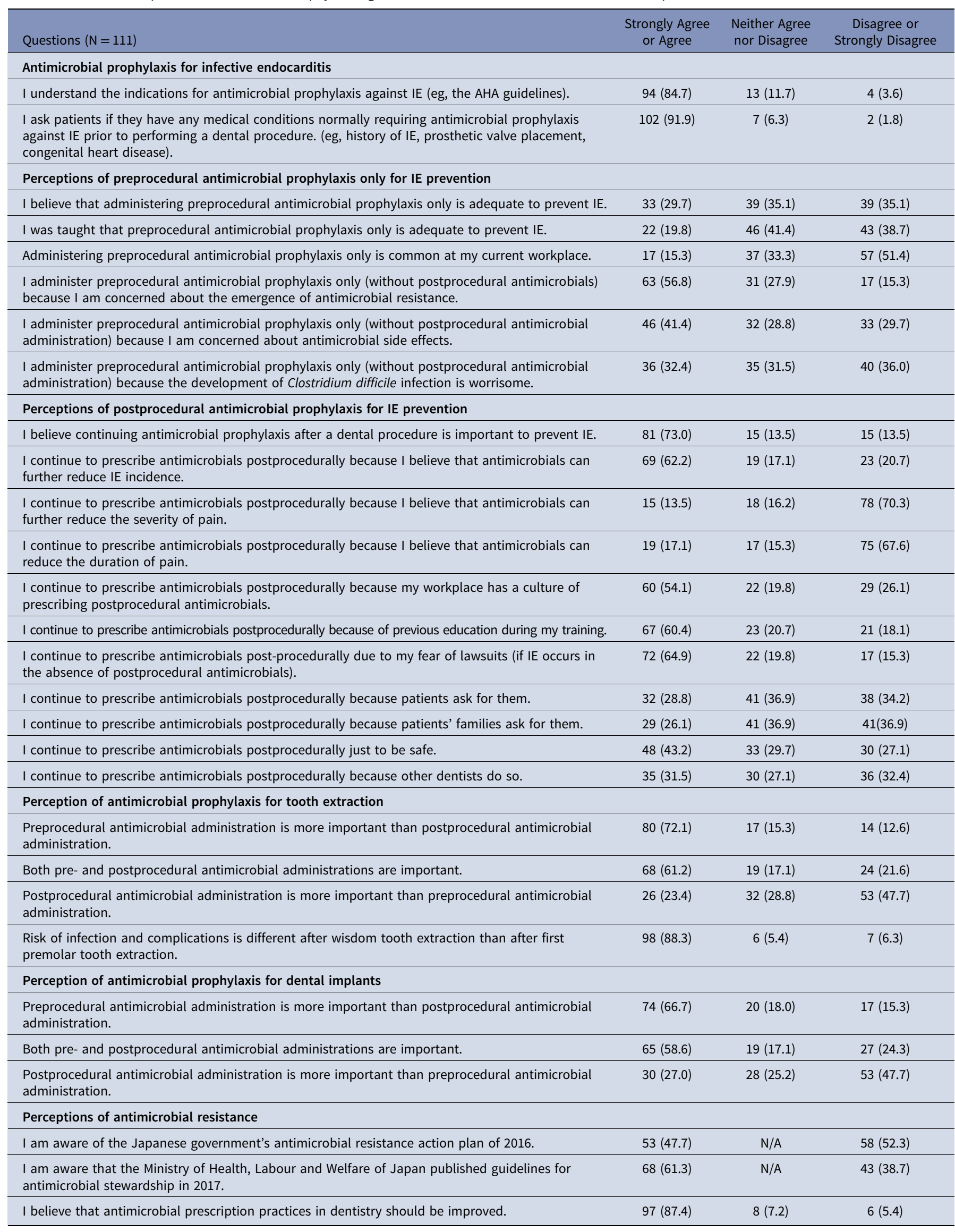

NOTE. IE; infective endocarditis, AHA; American heart association, N/A; not available. 
Table 1 shows the respondents' perceptions of antimicrobials prophylaxis. Regarding antimicrobial prophylaxis against IE, although $>80 \%$ of respondents reported that they understood the guidelines for the prevention of infective endocarditis, ${ }^{4,5}$ $>70 \%$ believed that continuing postprocedural antimicrobial administration was important for IE prevention, and $>80 \%$ reported having prescribed prophylactic antimicrobials against the current guidelines to patients with high-risk cardiac conditions in both the pre- and postprocedural periods (Appendix 2 online). ${ }^{4,5}$ Moreover, antimicrobial prophylaxis against IE was commonly administered to patients with other cardiac conditions, such as those with a pacemaker or history of percutaneous coronary intervention. The prescribing practices were influenced by the fear of a lawsuit (64.9\%), previous training in antimicrobial prophylaxis (60.4\%), and/or the prescribing culture at the respondents' current place of employment (54.1\%) (Table 1).

Regarding antimicrobial prophylaxis to prevent infections or complications following selected dental procedures in patients with no underlying illness, $81.1 \%$ of respondents reported that they did not prescribe antimicrobials for noninvasive procedures but did so for dental implant placements (95.5\%), wisdom tooth extractions (93.7\%), frenotomies (76.6\%), and orthodontic premolar tooth extractions (64.9\%) (Appendix 2 online). Although more than half the respondents considered pre- and postprocedural antimicrobial prophylaxis necessary to prevent infections or complications, they frequently prescribed antimicrobials only after a dental procedure (Appendix 2 online).

\section{Discussion}

The current study revealed that among dentists, behaviors dictating their prescription of antimicrobial prophylaxis against IE, local infections, and complications following common dental procedures varied widely. Moreover, respondents' self-reported behavior demonstrated that periprocedural antimicrobial overprescribing apparently stems from deeply entrenched misperceptions about the relevant pathophysiology, litigation fears, and prevailing culture despite putative familiarity with current recommendations against this behavior.

Most respondents reported understanding the current guidelines for IE prevention using antimicrobial prophylaxis but considered it important to continue postprocedural antimicrobial administration in contradiction to the guidelines. They also prescribed antimicrobial prophylaxis for non-high-risk cardiac conditions. Both the extended duration and expanded use of antimicrobial prophylaxis are likely to be associated with entrenched beliefs regarding periprocedural antimicrobial therapy. The current guidelines recommend antimicrobial prophylaxis only before a procedure in patients with a high-risk cardiac condition, such as cyanotic heart disease, a history of IE or prosthetic valve. ${ }^{4,5}$ However, in real-life settings, antimicrobial prophylaxis was frequently prescribed postprocedurally. ${ }^{3}$ The frequency of litigation related to dental procedures in Japan may be influencing dentists' prescribing behavior. ${ }^{8}$ In terms of the association between antimicrobial prescribing patterns and previous education or workplace culture, dentists' perception of antimicrobial prophylaxis did not differ significantly between hospital settings in this study (Appendix 3), suggesting that inadequate education and the failure to update antimicrobial practice in dentistry may be quite widespread in Japan.

Current evidence supports prophylactic antimicrobial use against local infections and complications following invasive dental procedures, such as tooth extractions and dental implantations. ${ }^{6,7}$ In this study, more than half of the respondents reported prescribing antimicrobials only after a dental procedure (Appendix 2 online). In principle, antimicrobial prophylaxis should be administrated before an invasive procedure. Moreover, continuing postprocedural administration to reduce further the risk of local complications or infections remains controversial. ${ }^{6,7}$ Previous studies have also cited the inappropriate continuation of postprocedural prophylaxis., ${ }^{3,9}$

This study has a number of limitations. The sample size was small, and selection bias may have occurred because the respondents were attendees of a regional academic conference. Moreover, their responses may not reflect their actual antimicrobial prescribing practices. Also, the questionnaire did not ask about the specific types of antimicrobial most frequently prescribed.

Dental antimicrobial prescribing practices for the prevention of IE, local infections, and complications following common dental procedures varied widely, and multiple factors influenced the decision to prescribe. Dentists often prescribed antimicrobials postprocedurally despite limited supporting evidence. Current practice patterns suggest that antimicrobials may be overprescribed in dentistry. Intervention is urgently needed to optimize antimicrobial prescription in dentistry.

Supplementary material. To view supplementary material for this article, please visit https://doi.org/10.1017/ice.2019.186.

Author ORCIDs. Hitoshi Honda, (D) https://orcid.org/0000-0002-4870-0730

Acknowledgments. We thank all the dentists who responded to the questionnaire. We are indebted to James R. Valera for his assistance in editing the manuscript.

Financial support. No financial support was provided relevant to this article.

Conflicts of interest. All authors report no conflicts of interest relevant to this article.

\section{References}

1. Suda KJ, Roberts RM, Hunkler RJ, Taylor TH. Antibiotic prescriptions in the community by type of provider in the United States, 2005-2010. J Am Pharm Assoc 2016;56:621-626.

2. Thompson W, Sandoe JA. What does NICE have to say about antimicrobial prescribing to the dental community? Br Dent J 2016;220:193-195.

3. Suda KJ, Henschel H, Patel U, Fitzpatrick MA, Evans CT. Use of antibiotic prophylaxis for tooth extractions, dental implants, and periodontal surgical procedures. Open Forum Infect Dis 2018;5(1):ofx250.

4. Wilson W, Taubert KA, Gewitz M, et al. Prevention of infective endocarditis. Circulation 2007;116:1736-1754.

5. Habib G, Lancellotti P, Antunes MJ, et al. 2015 ESC Guidelines for the management of infective endocarditis: The Task Force for the Management of Infective Endocarditis of the European Society of Cardiology (ESC). Eur Heart J 2015;36:3075-3128.

6. Lodi G, Figini L, Sardella A, Carrassi A, Del Fabbro M, Furness S. Antibiotics to prevent complications following tooth extractions. Cochrane Database Syst Rev 2012;11:CD003811.

7. Esposito M, Grusovin MG, Worthington HV. Interventions for replacing missing teeth: antibiotics at dental implant placement to prevent complications. Cochrane Database Syst Rev 2013;7:CD004152.

8. Judicial statistics 2018. Supreme court of Japan website. http://www.courts. go.jp/saikosai/vcms_lf/29052604sinryokakmoku.pdf. Published 2018. Accessed May 24, 2019.

9. Durkin MJ, Hsueh K, Sallah YH, et al. An evaluation of dental antibiotic prescribing practices in the United States. J Am Dent Assoc 2017;148: 878-886. 